\title{
Die aktuellen Lieferengpässe sind kein singuläres Ereignis
}

\author{
Ronald Bogaschewsky | Konstantin Sauer*
}

Die aktuellen Lieferengpässe kosten Milliarden und werden häufig einseitig auf Folgen der Corona-Pandemie zurückgeführt. Es wäre jedoch naiv, diese als singuläres Ereignis anzusehen und davon auszugehen, dass nach der Krise alles wieder bestens funktionieren würde. Es bedarf einer gesamtheitlichen Betrachtungsweise der Situation und der zu erwartenden Entwicklungen, die neben ökonomischen Aspekten auch Fragen der wachsenden Ressourcenknappheit, des Klimawandels, geostrategischer Herausforderungen und damit alternativer Strukturen der globalen Wertschöpfungsnetzwerke adressiert.

\section{Ursachenanalyse}

Bogaschewsky: Die Auftragsbücher sind voll, aber ob nun ElektronikChips oder Rohstoffe - die Versorgungslage ist extrem angespannt und vielerorts steht sogar die Produktion still. Es ist von Force Majeure die Rede, was letztlich heißen soll, dass niemand etwas dafürkönne. Angesichts einer kaum zu erwartenden Pandemie mag dies ja auch teilweise stimmen, aber eben nur teilweise. Wenn Lieferanten wegen Werksschließungen nicht produzieren oder Häfen die weiterzuleitenden Waren nicht abfertigen können, scheint es tatsächlich in kurzer Sicht kaum Reaktionsmöglichkeiten zu geben. Alternative Lieferwege sind in der Regel ebenfalls betroffen oder kommen aus Zeit- oder Kostengründen erst gar nicht infrage. Tatsächlich sind jedoch einige Unternehmen stärker betroffen als andere und dies durchaus auch in ein und derselben Branche. Bekanntermaßen leidet ein US-amerikanischer Elektroautohersteller erheblich weniger unter dem Chipmangel als seine Konkurrenten. Eine Ursache soll sein, dass dieser im Gegensatz zu vielen anderen in der Krise nicht sofort alle Lieferaufträge reduziert oder gar storniert hat. Partnerschaftliche und vertrauensvolle Beziehungen zu Lieferanten zahlen sich besonders, aber nicht nur in der Krise aus. Fakt ist auch, dass man über die Jahrzehnte hinweg häufig der Kosteneffizienz Priorität eingeräumt hat

Prof. Dr. Ronald W. Bogaschewsky | Lehrstuhl für BWL und Industriebetriebslehre | Julius-Maximilians-Universität Würzburg

Dr. Konstantin Sauer | Finanzvorstand und stell. Vorstandsvorsitzender | ZF Group 
- weil die Dynamik in der jeweiligen Industrie bzw. die direkten Konkurrenten dies vermeintlich so erzwangen. So hat man sich notgedrungen (,,halb zog es ihn, halb sank man hin“) in eine Effizienzsteigerungsspirale begeben, wo aus „schlank“ nicht selten „magersüchtig“ wurde. Dies ging auf Kosten der Resilienz, also der Fähigkeit, auf Dynamiken schnell zu reagieren und rasch wieder in einen stabilen und erfolgreichen Zustand zurückzukehren. Gleichzeitig nutzte man das weltoffene Wirtschaftssystem für die Globalisierung der Wertketten, wobei mehr oder weniger unbemerkt mehr oder weniger monopolistische Anbieterstrukturen entstanden. Dies gilt für viele Bereiche verarbeiteter (mineralischer) Rohstoffe und für weitere Grundstoffe sowie für Vorprodukte diverser Industrien und insbesondere auch für diverse Elektronikprodukte aus dem asiatischen Raum und insbesondere aus der VR China. Das Maß der Abhängigkeit zeigt sich in der Not - und die ist jetzt so groß wie seit sehr langer Zeit nicht mehr.

Sauer: Rohstoff- und Materialengpässe haben die globale Industrie fest im Griff. Auch die Automobilindustrie und deren Zulieferer sind massiv von den weltweiten Liefereinschränkungen betroffen. Besonders der unersättliche Hunger nach Halbleitern macht einigen Branchen zu schaffen. Derzeit existiert eine Verknappungssituation historischen Ausmaßes, die vor allem durch die reduzierte Nachfrage der gesamten Automobilindustrie während des Corona-bedingten Lockdowns im Jahr 2020 verursacht wurde. Da die Unterhaltungselektronikbranche zeitgleich mehr nachgefragt hat, wurden die vorhandenen Kapazitäten aufgezehrt, und der Aufbau zusätzlicher Volumina dauert an. Die Abhängigkeitsverhältnisse zwischen Lieferanten und Kunden sind in diesem Kontext eindeutig geklärt: Mit mageren zehn Prozent macht die Automobil- und deren Zuliefererindustrie nur einen Bruchteil des Einkaufsvolumens auf dem hart umkämpften Mikrochipmarkt aus. Die Konkurrenz aus Kommunikationsund Unterhaltungsindustrie ist wesentlich schwergewichtiger. Allein Smartphones verschlingen rund die Hälfte aller produzierten Halbleiter. Man kann es drehen und wenden wie man will: Mikroprozessoren für die Automobilindustrie sind mehr oder weniger ein Nischenprodukt für die international agierenden Produzenten. Hinzu kommen hohe Umrüstungszeiten, teils undurchlässige Lieferketten sowie die Nutzung veralteter Wafer-Technologien. Auch der Just-in-time-, bzw. Just-in-Sequence-Ansatz und die daraus resultierenden fehlenden Produktionspuffer haben die Lage innerhalb unserer Branche verschärft. Zudem zeigt der Blick in die Zukunft: Die Trends zur Mobilität und Elektrifizierung, zum autonomen Fahren und zur Digitalisierung werden den Bedarf an Mikroprozessoren und passiven Bauelementen noch weiter in die Höhe treiben. Während sich der Elektronikanteil des vernetzten, autonomen und elektrischen Autos in den nächsten fünf Jahren laut Prognosen mehr als verdoppeln soll, wird sich der Bedarf an Halbleitern voraussichtlich verdreifachen. Jedes Produkt, das die Zulieferindustrie nicht bedarfs-, zeit- oder qualitätsgerecht liefert, führt zu Produktions- und Lieferausfällen bei den Kunden. Schätzungen renommierter Consultingunternehmen gehen davon aus, dass dadurch allein im Jahr 2021 bis zu elf Millionen Fahrzeuge nicht produziert werden können. Auch ZF muss die aktuellen Lieferengpässe sowie eine Verteuerung von Rohstoffen wie Stahl, Aluminium, Magnesium oder Kunststoffen verkraften. Denn wir verändern unseren technologischen Fußabdruck mit Riesenschritten in Richtung Elektromobilität, autonomes Fahren und Digitalisierung. Fast alle unsere Produkte mit einem elektri- 
schen Steuergerät verwenden Halbleiter. Insofern sind Kameras, elektrische Lenkungen, Bremsen- und Getriebesteuergeräte davon betroffen, aber auch Schalthebel, Sicherheitsgurte, Steuergeräte für Elektromotoren oder Dämpfer. Der Reaktionsbedarf kommt dabei von zwei Seiten: zum einen von der Materialversorgung und zum anderen von unseren Kunden, die ihre Abrufe teils kurzfristig ändern. Diese Situation verlangt von uns ein Höchstmaß an Flexibilität und Einsatz innerhalb unserer Taskforces, um die Belieferung unserer Kunden stabil zu halten.

\section{Weitere und zukünftige Risiken}

Bogaschewsky: Bei Entspannung der Pandemielage und damit verbundener Disruptionen in den Wertschöpfungsketten dürfte sich mit Abarbeiten des Auftragsstaus nach und nach eine Normalisierung einstellen. Wenn man den Epidemiologen und Virologen folgt, wird der Corona-Virus jedoch nicht komplett zu eliminieren sein und auch vermutlich weiter mutieren. Ob die Bedrohung auf das Niveau „normaler“ Grippewellen, die bekanntlich bei stärkeren Ausbrüchen allein in Deutschland rund 25.000 Menschenleben gekostet haben, sinken wird, scheint bisher nicht abschließend beurteilt werden zu können. Vielen Analysten, die eine ganzheitlichere Sichtweise einnehmen und interdisziplinäre Risikobewertungen vornehmen, ist klar, dass Corona eine große, aber kaum die zentrale Herausforderung der nächsten Jahrzehnte darstellt. Diese liegt eindeutig in den Folgen des Klimawandels. Die Bedrohungen und Disruptionen aufgrund von klimatisch bedingten Ereignissen wie Dürren, Starkregen, Hochwasser und Stürmen in Verbindung mit Folgewirkungen wie Zerstörungen von Lebensräumen für Mensch und Tier, daraus resultierenden Flüchtlingsbewegungen sowie wirtschaftlichen Schäden ist kaum zu überschätzen. Die Daten von Rückversicherern und Geographen sprechen eine eindeutige Sprache. Entscheider in der Industrie sollten sich daher nicht wie Querdenker in der Pandemie verhalten, sondern - nach Jahrzehnten der Diskussion und Kommunikation wissenschaftlicher Erkenntnisse - endlich faktenbasiert und ernsthaft mit dem Thema beschäftigen. Erfreulicherweise entziehen große institutionelle Investoren mittlerweile zunehmend solchen Industrien die Finanzierung, die als CO2intensive Unternehmen für die weitere Zuspitzung der Klimabedingungen auf diesem Planeten stehen. Sowohl in der praktischen als auch in der wirtschaftswissenschaftlichen Analyse notorisch unterbelichtet scheint die Herausforderung der Verfügbarkeit nicht-regenerativer Ressourcen bzw. der daraus gewonnenen Roh- und Grundstoffe zu sein. Im betrieblichen Einkauf orientiert man sich nach wie vor primär am Markt, der bekanntlich vor allem die kurzfristige Angebots- und Nachfragesituation abbildet. Teilweise greift man mittels Futures oder anderer Instrumente ein wenig in die Zukunft. Nicht erst seit heute ist jedoch die systematische Berücksichtigung von sich abzeichnenden Knappheiten wesentlicher, für die Produktion benötigter Rohstoffe unabdingbare Voraussetzung für die Zukunftssicherung. Geologen und spezialisierte Institute wie die Deutsche Rohstoffagentur DERA stellen hier vielfältige Informationen zu Rohstoffen, deren Vorkommen, geographischen Verteilung, Reichweiten sowie Analysen zur Verfügung. Hierüber lassen sich direkt Risiken für die eigenen Wertschöpfungsketten ablesen. Leider gilt dies insbesondere für viele Rohstoffe, die für Zukunftstechnologien benötigt werden, beispielsweise bei der E-Mobilität, bei der Windkraft und der Fotovoltaik. „Der Markt wird 
es schon richten" erscheint in diesem Zusammenhang als sehr naive Idee. Wenig überraschend ist aus dem freiem Marktparadigma in Sachen Rohstoffe - dies gilt auch für Wasser - ein geostrategisches Spiel geworden, das offenbar bisher die EU und Deutschland nicht mitspielen (wollen). Die VR China hat bereits vor Jahren begonnen, sich nicht nur das Wasser in der Himalaya-Region zu sichern, sondern auch für viele Jahrzehnte die Ausbeutungsrechte von Minen in Afrika und Asien. Nicht erst seit der Krise um Schwere Seltene Erden sollte klar sein, dass dies nicht unbedingt getan wird, um die gewonnenen - und zumeist in China weiterverarbeiteten Rohstoffe auf dem freien Weltmarkt anzubieten, sondern um damit die eigenen inländischen Fertigungsstrukturen abzusichern. Genauso wie das Wasser des Himalayas eine überlebenswichtige Ressource für süd(ost)asiatische Länder darstellt, gilt dies für die Rohstoffe auf der Erde für unsere Produktionsbasis. Es wird Zeit, dass dieser Wake-up Call in den Unternehmen und bei den Entscheidungsträgern in der EU endlich vernommen wird und eigene Maßnahmen angegangen werden.

Sauer: Corona und der Klimawandel gehören zweifelsohne zu den wesentlichen Bedrohungen unserer Zeit. Durch die Pandemie hat sich das Tempo bei der Dekarbonisierung stark erhöht. So kündigen viele Staaten auf der ganzen Welt die Abkehr vom Fahrzeug mit Verbrennungsmotor an und setzen entsprechende Regelungen in Kraft, die den Markt heute bereits beeinflussen. Für ZF hat das unmittelbare Konsequenzen, denn wir müssen die zusätzlichen Investitionen in die Technologien der Elektromobilität beschleunigen, noch stärker priorisieren und uns auch strukturell verändern. Ein zweiter Aspekt ist allerdings mindestens ebenso bedeutend: die Veränderung der Arbeitswelt und der Prozesse durch die Digitalisierung. Vor zehn Jahren hätten sich Lockdowns vollkommen anders ausgewirkt und die Folgen wären weitaus gravierender gewesen. Wir haben als Unternehmen davon profitiert, dass wir seit Jahren kontinuierlich an der Digitalisierung arbeiten. Deshalb konnte ZF schnell und flexibel auf die Pandemie reagieren und neue Wege der virtuellen Zusammenarbeit etablieren - in technologischer, aber auch in menschlicher Hinsicht. Das spricht für unsere Anpassungsfähigkeit und das Engagement jedes Einzelnen. Disruptiven Ereignissen als Folge klimatischer Veränderungen müssen wir intelligente sowie nachhaltige Stellhebel entgegensetzen, um ihre Auswirkungen abzumildern. Klimaschutz ist ein zentrales Thema unserer Zeit und fester Bestandteil unserer ZF-Strategie „Next Generation Mobility“. Wir verfolgen damit einen agilen und integrierten Ansatz, um den globalen Herausforderungen angemessen zu begegnen und eine emissionsfreie, sozialgerechte und nachhaltige Mobilität gestalten zu können. ZF hat sich zum Ziel gesetzt, bis spätestens 2040 klimaneutral zu werden - und damit zehn Jahre früher als von der EU gefordert. Um dies zu erreichen, brauchen wir eine breitflächige Elektrifizierung über batterieelektrische und Hybridfahrzeuge. Aber das allein wird nicht genügen: wir müssen alle verfügbaren Register ziehen - auch, damit individuelle Mobilität erschwinglich bleibt. Dazu gehören E-Fuels und die Brennstoffzelle als strategische Mittel zur CO2-Reduzierung. Und zusätzlich ganz neue, disruptive Mobilitätskonzepte, wie zum Beispiel autonom fahrende Shuttles. Auch übernehmen wir Verantwortung für die Emissionsreduzierung in der Lieferkette. Wir wissen, dass ein starker Wandel vor uns steht - zum Beispiel setzen wir auf neue Technologien wie CO2-freien Stahl und den Aufbau einer Wasserstoff-Infrastruktur. Wir gehen davon aus, dass wir durch die intensive Zusammenarbeit mit unseren Lieferanten 
unsere Emissionen fast halbieren können. Warum hat ZF sich dieses Ziel gesetzt? Der Klimawandel erfordert ein schnelles und zielorientiertes Handeln. Wir wollen unseren Beitrag dazu leisten, den Temperaturanstieg auf deutlich unter zwei Grad zu begrenzen. Damit das erreicht wird, muss es der Weltgemeinschaft gelingen, den Ausstoß von $\mathrm{CO} 2$ zu reduzieren. Klimaneutrales Handeln kann nur durch gemeinsame Anstrengungen von Industrie, Staat und Gesellschaft erreicht werden. Voraussetzung ist ein europaweiter, langfristig stabiler und offener Regulierungsrahmen. Im Sinne eines verlässlichen Deals gilt: Das eine wird ohne das andere nicht funktionieren. Daher muss dieser Rahmen langfristig geplant und von allen Akteuren verbindlich umgesetzt werden. Wenn wir einen breiten technologischen Ansatz wählen, wenn Politiker und Ingenieure gemeinsam Lösungen erarbeiten, steigen die Chancen, dass die Transformation der Automobilbranche und seiner Wertschöpfungsketten klimapolitisch, industriepolitisch und auch gesellschaftspolitisch ein Erfolg wird.

\section{Mögliche Maßnahmen}

Bogaschewsky: Die Diskussion um die Umstrukturierung der globalen Wertschöpfungsketten ist seit geraumer Zeit im Gange. Aus unternehmensstrategischer Perspektive sind vielerorts die gestiegenen Risiken zumindest wahrgenommen worden, während konkret umgesetzte, umfassendere Maßnahmen bisher nur selten sichtbar sind. Im Einkauf sind viele zwecks Erhöhung der Resilienz mit intensivierter Beschaffungsmarktforschung sowie der Identifizierung und Analyse alternativer Lieferquellen beschäftigt, wobei das Ziel oftmals in der Streuung der Risiken über unterschiedliche Geographien liegt. Dies scheint zum überwiegenden Teil eine Reaktion auf die aktuellen Lieferengpässe infolge der Pandemie oder auch kritischen Ereignissen wie die Havarie-bedingte Blockade des Suezkanals zu sein. Das Supply Chain-bezogene Risikomanagement läuft damit immer noch meistens der realen Entwicklung hinterher und reagiert, anstatt proaktiv zu agieren. Ein strategisches Risikomanagement der Versorgungsketten unter Beachtung einer Vielzahl von sich gegenseitig beeinflussenden Faktoren wie klimabedingte Risiken, politische Entwicklungen, Flüchtlingsbewegungen, Verfügbarkeit natürlicher Ressourcen und letztlich globale Gesundheit (ein weit über die aktuelle Pandemie hinausgehendes Themenfeld) in Verbindung mit Armut und Hunger erscheint für zukunftssichere Wertschöpfungssysteme unverzichtbar. Die Zeiten, wo Unternehmen auf der Jagd nach den primär kostengünstigsten Standorten und Lieferquellen waren, dürften sich dem Ende nähern. Zudem bekommen CO2-Steuern innerhalb von Ländern und Wirtschaftsräumen zunehmendes Gewicht bei der Beurteilung der wirtschaftlichen Vorteilhaftigkeit alternativer Fertigungsstrukturen. Unternehmen müssen verstärkt unterschiedliche globale Entwicklungsszenarien bei ihren strategischen Entscheidungen zum Supply Chain Design berücksichtigen, da sich viele Parameter fundamental und rasch verändern bzw. bereits verändert haben. Daraus dürften sich Vorteilhaftigkeiten gegenüber Entscheidungen aus der Vergangenheit verschieben. Eine dieser Veränderungen muss in der deutlichen Reduzierung von massiven Abhängigkeiten bei der Versorgung mit wesentlichen Grundstoffen und Produkten liegen. Elektronische Bauteile, Chips und Software sind hierfür ebenso Beispiele wie lebenswichtige pharmazeutische Produkte. Dies kann häufig nur mittels Aufbaus neuer Produktionsstätten und Lieferquellen in vergleichsweise 
„sicheren“ Geographien erreicht werden und erfordert strategisch weitsichtige Investitionen. Das Gelingen hängt u.a. von gemeinschaftlichen Anstrengungen ganzer Industrien sowie dem Aufsetzen geeigneter politischer Unterstützungsmaßnahmen ab. Viel Zeit bleibt hierfür nicht.

Sauer: Die derzeitige Verknappungssituation ist beispiellos. Aufgrund der geringen Verfügbarkeiten an Bauteilen, Materialien und Rohstoffen haben wir höhere Lagerbestände aufgebaut, um unsere Produktion jederzeit abzusichern. Diese außergewöhnliche Situation kann jedoch mittel- bis langfristig nicht ausschließlich mit einem Ruf nach mehr Lagerhaltung beantwortet werden, sondern stattdessen mit einer angepassten intelligenten Beschaffungsstrategie. Dieses bedeutet auch eine stärkere Regionalisierung der Lieferungen, unter anderem auch durch neue Ursprungsregeln in Handelsabkommen wie dem NAFTA-Nachfolgeabkommen USMCA. Wir setzen bereits heute verstärkt auf lokale Lieferketten. Das heißt, unsere Zulieferbetriebe befinden sich in jenen Ländern, in denen wir für unsere Kunden produzieren. Engpasssituationen begegnen wir jedoch nicht nur durch eine stärkere Regionalisierung, sondern auch durch die Suche nach alternativen Bezugsquellen in anderen Produktionsländern. So durchforsten beispielsweise unsere ZF-Gusslieferanten, die dringend auf die Versorgung mit Magnesium angewiesen sind, die globalen Märkte nach freien Kapazitäten. Aufgrund des hohen chinesischen Marktanteils von über 85 Prozent ist die Flexibilität, die Mindermengen aus dem chinesischen Markt komplett zu kompensieren, jedoch eingeschränkt. Um von nationalen Krisen und internationalen Handelseinflüssen unabhängig zu sein, haben wir seit langem ein engmaschiges Netz an Produktions- und Logistikstrukturen geknüpft, um auch kurzfristig auf alternative Liefermöglichkeiten, Transport- und Beschaffungswege zurückgreifen zu können. Die Herausforderungen der Corona-Pandemie haben unsere ZFStrategie grundsätzlich bestätigt. Wir werden zukünftig noch intensiver daran arbeiten, alternative Lieferanten vorzuhalten. Auch produktseitig haben wir Möglichkeiten gegenzusteuern. So sind wir in der Lage, alternative Prozessoren zu verwenden - vorausgesetzt, sie sind verfügbar und haben exakt die geforderten Maße. In der Regel handelt es sich dabei um Chips mit etwas kleinerer Speicherkapazität und weniger Rechenleistung. Wenn der Kunde die Zahl der gewünschten Softwarepakete reduziert, kann er somit mit der schlankeren Alternative bedient werden. Damit konnten wir bereits an einigen neuralgischen Stellen Lieferketten stabil halten. Es fordert allerdings die Entwickler heraus, weil sie umdenken müssen. Gemeinsam mit unseren Kunden definieren wir, ob alle Softwarepakete gebraucht werden. Der Aufwand ist nicht unerheblich, lohnt sich aber, denn wir können so weiter für unsere Kunden produzieren. Auch die Vernetzung mit Partnern aus benachbarten Branchen über ganze Lieferketten hinweg ist ein wichtiger Ansatz. So gehört ZF zu den Gründungsunternehmen des Partner-Netzwerks Catena-X, der Allianz für sicheren und unternehmensübergreifenden Datenaustausch in der Fahrzeugindustrie. Einheitliche Daten entlang der gesamten Wertschöpfungskette ermöglichen uns zum Beispiel, Bauteile und Software zurückzuverfolgen angefangen vom Rohstofflieferanten, über Teile-, Komponenten-, Systemund Modullieferanten bis hin zum Fahrzeughersteller. Auch Händlernetzwerke, Logistikanbieter sowie Verwerter sind hier einbezogen. Das hilft uns beispielsweise dabei, die Versorgung mit wichtigen Bauteilen sicherzustellen. Auch können wir künftig Lieferketten transparenter gestalten und somit anspruchsvolle Nachhaltigkeitskriterien erfüllen. Das 
gilt genauso für Sozial- oder Menschenrechtsstandards wie auch für die Reduktion des $\mathrm{CO} 2-$ Fußabdrucks in Produktion und Logistik. Um es abschließend auf den Punkt zu bringen: Fahrzeughersteller und Tier-1-Zulieferer müssen ihre traditionellen Beschaffungs- und Entwicklungstools anpassen und neue Fähigkeiten aufbauen. Sie müssen ihre Position in der Wertschöpfungskette bei Halbleiter- und Elektronikhardware neu definieren, um einen Wettbewerbsvorteil zu schaffen. Und es gilt, Partnerschaften in den Bereichen Elektronik, Halbleiter und Software entlang der gesamten Supply Chain auf- und auszubauen. All dies sind mögliche Ansatzpunkte, um aus der Misere rund um die grassierende Versorgungskrise zu lernen. 